\title{
Patient reported outcome measures (PROMs) in primary care: an observational pilot study of seven generic instruments
}

\author{
Jan-Willem Weenink*, Jozé Braspenning and Michel Wensing
}

\begin{abstract}
Background: Patient reported outcome measures (PROMs) have been introduced in studies to assess healthcare performance. The development of PROMs for primary care poses specific challenges, including a preference for generic measures that can be used across diseases, including early phases or mild conditions. This pilot study aimed to explore the potential usefulness of seven generic measures for assessing health outcomes in primary care patients.

Methods: A total of 300 patients in three general practices were invited to participate in the study, shortly after their visit to the general practitioner. Patients received a written questionnaire, containing seven validated instruments, focused on patient empowerment (PAM-13 or EC-17), quality of life (EQ-5D or SF-12), mental health (GHQ-12), enablement (PEI) and perceived treatment effect (GPE). Furthermore, questions on non-specific symptoms and number of GP contacts were included. After 4 weeks patients received a second, identical, questionnaire. Response and missing items, total scores and dispersion, responsiveness, and associations between instruments and other measures were examined.
\end{abstract}

Results: A total of 124 patients completed the questionnaire at baseline, of whom 98 completed it both at baseline and 4 weeks later (response rate: $32.7 \%$ ). The instruments had a full completion rate of $80 \%$ or higher. Differences between baseline and follow up were significant for the EQ-5D $(p=0.026)$, SF-12 PCS $(p=0.026)$ and the GPE $(p=0.006)$. A strong correlation ( $r \geq 0.6)$ was found between the SF-12 MCS and GHQ-12, at both baseline measurement and after four weeks. Other observed associations between instruments were moderately strong. No strong correlations were found between instruments and non-specific symptoms or number of GP contacts.

Conclusions: The present study is among the first to explore the use of generic patient-reported outcome measures in primary care. It provides several leads for developing a generic PROM questionnaire in primary care as well as for potential limitations of such instruments.

Keywords: Patient-reported outcome measures, Primary care, General practice, Quality of life, Patient empowerment, Patient enablement

\footnotetext{
* Correspondence: janwillemweenink@gmail.com

Scientific Institute for Quality of Healthcare, Radboud University Medical

Center, P.O. Box 9101, 6500 HB Nijmegen, the Netherlands
} 


\section{Background}

Patient reported outcome measures (PROMs) are standardised, validated questionnaires that are completed by patients to measure perceived health status, functional status or health-related quality of life [1]. While PROMs are used in health research to document health outcomes, in particular treatment effectiveness in clinical trials [2], today they are also used to measure healthcare quality. For instance, in 2009 the National Health System in the UK started to use PROMs to assess the quality of four elective procedures [3]. The adoption of PROMs in primary care, however, poses specific challenges that are related to the specific characteristics of their patient population. Primary care patients show a wide range of diseases, including many early undifferentiated stages and mild conditions. Furthermore, primary care provides comprehensive and continuing healthcare [4]. From the WONCA competencies and corresponding characteristics of general practice follow some other domains that can be measured at the patient level and may be appropriate as outcome measures. These are that the general practitioner should "develop a person centred approach orientated to the individual, his/her family, their community, where it as important to understand how the patient copes with and views their illness as dealing with the disease process itself"; and that the general practitioner should "promote patient empowerment" [4]. Scales measuring patient enablement and patient empowerment may be appropriate to measure these domains. When developing PROMs for primary care these factors should be taken into account, implying that generic measures that can be used across diseases are preferable to disease-specific measures, and that a broad set of domains of general practice should be addressed by a PROM.

Many questionnaires exist that aim to assess primary care performance from the patients' perspective. For instance, the Primary Care Assessment Survey (PCAS) studies seven domains of general practice, such as accessibility, continuity, comprehensiveness and interpersonal treatment [5]. The European Task Force on Patient Evaluations of General Practice (EUROPEP) measures patient evaluations of a broad range of specific aspects of general practice care, such as showing interest, involving the patient in decision making and thoroughness [6], and the Patient Assessment of Chronic Illness Care (PACIC) studies chronic care delivery [7]. Most existing questionnaires for assessing primary care performance, however, focus on the organisation and process of healthcare delivery, instead of care outcomes.

Some validated questionnaires for functional status or quality of life, which were not primarily developed for primary care performance measurement, may be good options for PROMs in primary care. Before embarking on the development of a new tool, we explored a number of existing measures that focus on these domains in a pilot study. Besides its generic character, we felt that a potentially useful PROM should have high relevance for primary care patients (indicated by good response rates), have potential to discriminate between care providers (indicated by absence of highly skewed distributions), show responsiveness to change of patients' symptoms over time, and be predictably correlated with other measures. Based on these predefined criteria, we aimed to explore the potential usefulness of seven generic patient reported outcome measures in primary care. The results of this pilot study can possibly be used to inform further research and development of PROMs in primary care as well as for reflection on the potential limitations of PROMs in primary care.

\section{Methods}

\section{Design and setting}

An observational study was performed in patients who visited their general practitioner for consultation in one of three participating practices (five general practitioners in total). A maximum of 60 patients per general practitioner was invited to minimize workload for general practitioners. Practices were situated in the south-eastern part of The Netherlands, and concerned one practice in an urban area and two in a rural area. One practice was single-handed and two were group practices. Ethical approval was received for this study from the ArnhemNijmegen ethical committee.

\section{Study population}

A total of 300 patients was invited who visited one of the participating general practitioners for a consultation. Patients were not invited to participate if they were younger than 18 years old, terminally ill, or had psychological problems or a mental handicap as a result of which the GP estimated the patient was not suitable to participate in research at the moment. Written questionnaires were handed out by the general practitioner during the consultation. Patients were asked to complete the questionnaire and return it to the research institute in a prepaid envelope. In the questionnaire, patients were asked if they were willing to complete a second identical questionnaire after 4 weeks. If so, patients were sent a second questionnaire by the research institute.

\section{Measures}

We performed a comprehensive search in PubMed using keywords primary care and patient reported outcomes. We scanned articles and references of relevant articles for existing questionnaires on the outcome domains listed in Table 1. Furthermore, we consulted colleagues to identify instruments they had previously used. We searched the internet for a Dutch translation of questionnaires, and only included questionnaires that were available in Dutch. 
Table 1 Possible outcome domains for PROMS following from the WONCA definition of general practice

\begin{tabular}{lll}
\hline WONCA competency & WONCA characteristics & Possible outcome domain \\
\hline Comprehensive approach & Promotes health and well-being & General-, physical and mental health \\
Person-centred care & Promotes patient empowerment & Patient empowerment \\
& Centred on patient and context & Patient enablement \\
\hline
\end{tabular}

For some domains multiple questionnaires were found. We excluded questionnaires on the basis of length. The selected questionnaires are listed in Table 2, and are further elucidated on in the paragraphs below. Excluded questionnaires included the Measure Yourself Medical Outcome Profile (MYMOP) [8] and the Outcome Related Impact on Daily Life (ORIDL) [9] for unavailability of a Dutch translation, and the Sickness Impact Profile (SIP) [10] due to its length. Furthermore, the Spielberger StateTrait Anxiety Inventory (STAI) [11] was not included since it focuses specifically on anxiety. We chose to include generic instruments focusing on mental health, thereby limiting the total length of our questionnaire. Finally, we included a Global Perceived Effect scale (GPE) for assessing the effect of received care.

The Patient Activation Measure (PAM-13) and the Effective Consumer Scale (EC-17) were alternately used to measure patient empowerment. The PAM-13 consists of 13 items that evaluates a patient's knowledge, skills and confidence to manage their own health [12]. Item scores are converted in one activation score, reflecting a patient's activation level. Missing values are accounted for in calculation of the total score. The EC-17 consists of 17 items on 5 subscales (use of health information, clarifying priorities, communication with others, negotiating own role, and taking action) [13]. Item scores are converted to a score on a 0-100 scale. If more than 3 items are missing no total score is computed. Because the EC-17 specified having a disease in its questions, we added a not applicable response option, which we treated as missing data in computing the total score.

The EuroQol-5D (EQ-5D) and the Short Form 12 (SF-12) were alternately used to measure quality of life. The EQ-5D consists of five dimensions (mobility, self-care, usual activities, pain/discomfort and anxiety/depression)

Table 2 Domains and used questionnaires

\begin{tabular}{ll}
\hline Domain & Questionnaire \\
\hline Patient Empowerment & Patient Activation Measure (PAM-13) \\
& Effective Consumer Scale (EC-17) \\
General Health & EuroQol 5D (EQ-5D, including EQ-VAS) \\
& Short Form 12 (SF-12) \\
Mental Health & General Health Questionnaire (GHQ-12) \\
Enablement & Patient Enablement Instrument (PEI) \\
Effect & Global Perceived Effect (GPE) \\
\hline
\end{tabular}

with three response categories (no problems, some problems, extreme problems) [14]. Total score was calculated using Dutch population norms [15]. Furthermore, the EQ-5D contains a visual analogue scale (VAS) on which respondents score their current health status on a scale from 0 to 100 . The SF-12 is a 12 -item questionnaire measuring eight domains: physical functioning, role-physical, bodily pain, general health, vitality, social functioning, role-emotional and mental health [16]. Each item is scored on a 3- or 5-point Likert scale, and for each domain a total score is computed on a 0-100 scale. With these item scores, a physical component summary (PCS) and a mental component summary (MCS) can be calculated.

The General Health Questionnaire (GHQ-12) was used to measure mental health. It consists of 12 items, each with a 4-point response category (from 'better than usual' to 'much less than usual') [17], in which each item receives a score of $0,1,2$ or 3 . The total score for the GHQ-12 thus ranges from 0 to 36 , where a lower score reflects better mental health.

The Patient Enablement Instrument (PEI) was used to measure patient enablement [18]. It consists of six items, each with three response categories $(0=$ 'same or worse', 1 = 'better', 2 = 'much better'). The range of the aggregated sum score is 0 to 12 , with a higher score indicating a higher level of enablement.

A Global Perceived Effect (GPE) scale was used to measure perceived effect of treatment. The scale consists of one item that asks patients about perceived effect of treatment [19]. Patients score on a seven point response scale (with 1 = 'worse than ever', 7 = 'fully recovered'). Furthermore, we dichotomized the GPE scores into "improved" ("completely recovered" and "much improved") versus "not improved" ("slightly improved", "not changed", "slightly worsened", "much worsened", "worse than ever"), and added a question about treatment satisfaction (also on a seven-point scale).

The questionnaire also included questions about nonspecific symptoms and the number of GP contacts in the previous 12 months. Non-specific symptoms included fatigue, dizziness, headache, weakness, palpitation and sleep problems, and their presence can indicate underlying changes in emotional well-being [20]. As said, some instruments that aimed to measure the same domain were used alternately to reduce length. Therefore, a total of four versions of the questionnaire were used. 


\section{Sample size calculation}

For our explorative study, no figures were available for a proper power calculation given the new context of the questionnaires distributed. We based our sample size largely on what number of questionnaires would be feasible in terms of burden for the recruiting GPs and what sample size would give a meaningful precision. Therefore, we invited a total of 300 patients (60 per GP). We expected a response rate of approximately $50 \%$, resulting in all instruments having a response of 150 patients or 75 patients (in case of the EQ-5D, SF-12, PAM-13 and EC-17). This results in a precision (half width of the $95 \%$-CI for mean difference) of 4.8 and 3.5 points (assuming a 100 points scale with a SD of at most 15), which seemed sufficiently precise to detect non-small differences [21].

\section{Data-analysis}

We first studied the response on individual instruments, and the missing values on items. Instruments with a low response or a high number of missing scores were considered less appropriate for potential use in practice.

Secondly, we studied statistical dispersion of scores for each of the instruments, exploring mean, minimum and maximum scores and standard deviations. We examined if data was normally distributed by exploring histograms and using the Shapiro-Wilk test. Furthermore, we studied floor and ceiling effects in terms of percentage of patients using the most extreme (upper or lower) response categories. Instruments with a squeezed distribution, or high presence of floor and ceiling effects were thought to be less appropriate for potential use in practice.

Responsiveness has been defined as the ability of an instrument to accurately detect change when it has occurred [22]. Change in instrument scores between baseline and follow-up were explored, and were tested on significance with a paired samples t-test or in case of a skewed distribution with a Wilcoxon signed-rank test. We explored changes in scores between baseline and follow up, where positive changes could reflect the relief of complaints at baseline due to treatment or favourable natural development. Furthermore, we used Pearson correlation to identify moderate $(\mathrm{r}=.40-.59)$, strong $(\mathrm{r}=.60-.79)$ and very strong associations $(\mathrm{r}=.80=1.0)$ between instrument scores [23]. We explored if scores of instruments focusing on the same domains correlated.

Finally, we looked at treatment satisfaction, presence of non-specific symptoms and number of contacts with the GP to assess construct validity. Construct validity refers to the extent to which scores on a particular instrument relate to other measures in a manner that is consistent with theoretically derived hypotheses concerning the concepts that are being measured [24,25]. We expected that treatment satisfaction would be positively correlated with instruments scores [26], while the presence of non-specific symptoms was expected to have a negative correlation with instrument scores [20]. Furthermore, we explored if visiting frequency was associated with instruments scores. Since all seven instruments had previously been validated in other contexts, we expected that content validity was assured.

Finally, an a posteriori sample size calculation was performed to learn about the number of questionnaires needed to show a meaningful difference.

\section{Results}

Of 300 invited patients, 124 completed the questionnaire at baseline and 98 patients completed the questionnaire both at baseline and after 4 weeks (response rate: 32.7\%). Response percentages ranged from $16.7 \%$ to $50.0 \%$ across the participating general practitioners. Table 3 provides descriptive information of the study population. In comparison with Dutch GP population, our study population was less ethnically diverse and more likely to have one or more chronic illnesses [27]. Most prevalent chronic illnesses were cardiovascular disease (31.6\%), diabetes (15.3\%) and depressive symptoms (11.2\%). 56\% of patients with a chronic illness used medication.

\section{Response and missing items}

Response percentages and the number of missing items on the individual instruments are presented in Table 4. The response on the different instruments ranged from $87.5 \%$ to $99.0 \%$. Of each instrument, over $80 \%$ was completed without any missing items. The EC-17 had a relatively high number of missing values. The response on the EC-17 was $91.8 \%$, but because we treated the not applicable response option as missing and no total score was computed with more than 3 missing items, a total of 36 scores remained (73.5\%).

\section{Dispersion}

The median, minimum and maximum scores, as well as inter-quartile ranges (IQR) at baseline and after four weeks are presented in Table 5. Floor and ceiling effects for the specific measures are provided in Table 6. In comparison to other instruments, the EQ-5D had a high

Table 3 Patients' characteristics $(\mathbf{n}=98)$

\begin{tabular}{ll}
\hline & Study population \\
\hline Mean age (SD) & $62.5(12.2)$ \\
Percentage women & $44.2 \%$ \\
Percentage with higher education & $34.7 \%$ \\
Percentage with single household & $18.3 \%$ \\
Percentage of Dutch decent & $99.0 \%$ \\
Percentage with one or more chronic illnesses & $55.1 \%^{\mathrm{a}}$ \\
\hline
\end{tabular}

ancluded asthma, cardiovascular disease, COPD, depression and diabetes. 
Table 4 Response on individual measures

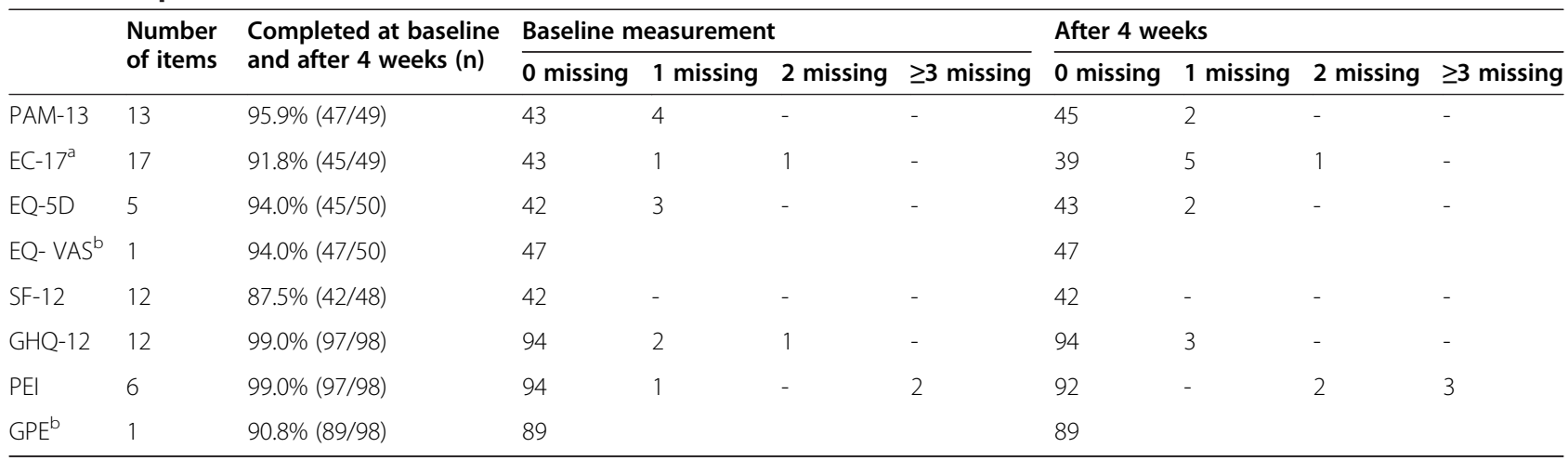

${ }^{a}$ If 3 or more items were missing or not applicable no total score was computed; 36 total scores computed (73.5\%).

${ }^{\mathrm{b}}$ Instrument consists of 1 item.

prevalence of maximum scores and the PEI had a high prevalence of minimum scores.

\section{Responsiveness}

All measures showed increased mean scores over time, indicating improvement in health status, though for most instruments no median score differences were observed. The differences in mean scores between baseline and follow up at four weeks were significant for the EQ-5D $(\mathrm{p}=0.026)$, SF-12 PCS $(\mathrm{p}=0.026)$ and the GPE $(\mathrm{p}=$ 0.006). When looking at dichotomous scores of the GPE, we found that 15 out of 89 patients at baseline right after the consultation, and 27 out of 89 patients after four weeks indicated to have improved after their visit to the GP. A total of 18 patients improved after four weeks in relation to baseline, while 6 patients worsened in these four weeks. Table 7 presents the percentage of patients that had an increased or worsened score for the specific measures. In comparison to other measures, the EQ-5D and PEI showed little change across time with approximately half of the patients having the same score at follow-up.

\section{Associations between instruments}

For baseline scores, strong associations were found between the SF-12 MCS and the GHQ $(r=-0.768, p=$ $0.000)$, and the EQ-5D en EQ-VAS $(r=0.604, p=0.000)$. Moderate associations were found between the PAM-13 and EQ-5D $(\mathrm{r}=0.409, \mathrm{p}=0.043)$, the PAM-13 and SF-12 MCS $(r=0.438, p=0.079)$, and the EQ-VAS and GPE ( $r=$ $0.429, \mathrm{p}=0.004)$. For scores after four weeks, strong associations were found between the EC-17 and EQ-VAS ( $r=$ 0.709, $\mathrm{p}=0.010)$, EQ-VAS and GPE $(\mathrm{r}=0.661, \mathrm{p}=0.000)$, the GHQ and SF-12 MCS $(r=-0.705, p=0.000)$, and the GHQ and EQ-VAS $(r=-0.633, p=0.000)$. Moderate associations were found between the PAM-13 and EQ-5D ( $\mathrm{r}=$ $0.440, \mathrm{p}=0.028)$, PAM-13 and EQ-VAS $(\mathrm{r}=0.552, \mathrm{p}=$ 0.003), PAM-13 and GPE $(r=0.481, p=0.001), E Q-5 D$ and EQ-VAS $(\mathrm{r}=0.568, \mathrm{p}=0.000), \mathrm{EQ}-5 \mathrm{D}$ and GPE $(\mathrm{r}=$ $0.542, \mathrm{p}=0.000), \mathrm{EC}-17$ and GHQ $(\mathrm{r}=-0.492, \mathrm{p}=0.002)$, GHQ and SF-12 PCS $(r=-0.420, p=0.006)$. When looking at change of scores on the instruments, strong associations were found between the EC-17 and EQ-VAS ( $r=0.554$, $\mathrm{p}=0.061), \mathrm{SF}-12 \mathrm{PCS}$ and SF-12 MCS $(\mathrm{r}=-0.523, \mathrm{p}=$

Table 5 Total scores and change of scores on individual measures $(\mathbf{n}=98)$

\begin{tabular}{|c|c|c|c|c|c|c|c|c|c|c|c|c|c|}
\hline \multirow[b]{2}{*}{ Instrument (n) } & \multirow[b]{2}{*}{$\begin{array}{l}\text { Theoretical } \\
\text { range of scale }\end{array}$} & \multicolumn{4}{|c|}{ Baseline score } & \multicolumn{4}{|c|}{ Score after four weeks } & \multicolumn{4}{|c|}{ Score difference } \\
\hline & & Median & Min. & Max. & IQR & Median & Min. & Max. & IQR & Median & Min. & Max. & IQR \\
\hline PAM-13 (47) & $0-100$ & 56.4 & 41.7 & 100.0 & $49.9-70.8$ & 56.4 & 40.1 & 100.0 & $49.9-70.8$ & 0.0 & -43.6 & 38.9 & -2.8 to 6.7 \\
\hline $\mathrm{EC}-17(36)^{\mathrm{a}}$ & $0-100$ & 76.5 & 55.9 & 100.0 & $75.0-83.8$ & 80.3 & 63.2 & 98.5 & $75.0-88.1$ & 0.0 & -10.9 & 36.8 & -2.4 to 4.4 \\
\hline EQ-5D (45) & $0-1$ & 0.84 & 0.15 & 1.00 & $0.79-0.89$ & 0.84 & 0.25 & 1.00 & $0.81-1.0$ & $0.00^{c}$ & -0.44 & 0.28 & 0.00 to 0.11 \\
\hline EQ- VAS (47) & $0-100$ & 70.0 & 30 & 100 & $60.0-80.0$ & 75.0 & 40 & 100 & $70.0-80.0$ & 0.0 & -25.0 & 20.0 & -5.0 to 10.0 \\
\hline SF-12 PCS (42) & $0-100$ & 42.3 & 18.7 & 60.5 & $32.4-53.5$ & 49.8 & 17.8 & 58.9 & $37.5-54.4$ & $2.0^{c}$ & -15.3 & 28.8 & -1.3 to 7.1 \\
\hline SF-12 MCS (42) & $0-100$ & 56.0 & 17.3 & 65.8 & $48.7-59.0$ & 54.1 & 19.8 & 62.7 & $49.3-58.9$ & 0.0 & -25.8 & 20.9 & -6.3 to 4.4 \\
\hline GHQ-12 (97) & $0-36^{b}$ & 9.0 & 3.0 & 26.0 & $7.0-13.0$ & 8.0 & 3.0 & 25.0 & $7.0-12.0$ & 0.0 & -13.0 & 10.0 & -2.0 to 2.0 \\
\hline PEI (97) & $0-12$ & 0.0 & 0.0 & 6.0 & $0.0-2.5$ & 0.0 & 0.0 & 12.0 & $0.0-2.0$ & 0.0 & -6.0 & 8.0 & -0.5 to 0.0 \\
\hline GPE (89) & $1-7$ & 4.0 & 2 & 7 & $4.0-5.0$ & 4.0 & 3 & 7 & $4.0-6.0$ & $0.0^{c}$ & -3.0 & 3.0 & 0.0 to 1.0 \\
\hline
\end{tabular}

${ }^{a}$ If 3 or more items missing then no score was computed.

${ }^{b}$ Lower score means better health.

${ }^{c}$ Mean score difference significant with $\mathrm{p} \leq 0.05$. 
Table 6 Floor and ceiling effects

\begin{tabular}{lllll}
\hline Instrument $(\mathbf{n})$ & Minimum score baseline & Maximum score baseline & Minimum score follow up & Maximum score follow up \\
\hline PAM-13 (47) & $0.0 \%(0 / 47)$ & $2.1 \%(1 / 47)$ & $0.0 \%(0 / 47)$ & $2.1 \%(1 / 47)$ \\
EC-17 (36) & $0.0 \%(0 / 36)$ & $2.8 \%(1 / 36)$ & $0.0 \%(0 / 36)$ & $0.0 \%(0 / 36)$ \\
EQ-5D (45) & $0.0 \%(0 / 45)$ & $22.2 \%(10 / 45)$ & $0.0 \%(0 / 45)$ & $44.4 \%(20 / 45)$ \\
EQ- VAS (47) & $0.0 \%(0 / 47)$ & $2.1 \%(1 / 47)$ & $0.0 \%(0 / 47)$ & $6.4 \%(3 / 47)$ \\
SF-12 PCS (42) & $0.0 \%(0 / 42)$ & $0.0 \%(0 / 42)$ & $0.0 \%(0 / 42)$ & $0.0 \%(0 / 42)$ \\
SF-12 MCS (42) & $0.0 \%(0 / 42)$ & $0.0 \%(0 / 42)$ & $0.0 \%(0 / 42)$ & $0.0 \%(0 / 42)$ \\
GHQ-12 (97) & $0.0 \%(0 / 97)$ & $0.0 \%(0 / 97)$ & $0.0 \%(0 / 97)$ & $0.0 \%(0 / 97)$ \\
PEI (97) & $54.6 \%(53 / 97)$ & $0.0 \%(0 / 97)$ & $60.8 \%(59 / 97)$ & $1.0 \%(1 / 97)$ \\
GPE (89) & $1.1 \%(1 / 89)$ & $5.6 \%(5 / 89)$ & $0.0 \%(0 / 89)$ & $6.7 \%(6 / 89)$ \\
\hline
\end{tabular}

0.000), and GHQ and SF-12 MCS ( $\mathrm{r}=-0.530, \mathrm{p}=0.000)$. A moderate association was found between the EQ-5D and EQ-VAS $(\mathrm{r}=0.438, \mathrm{p}=0.003)$.

\section{Associations with other measures}

At baseline, a total of 77 out of 91 patients reported to be very or absolutely satisfied with treatment (84.6\%). At follow-up this was 69 patients (75.9\%). A moderate positive correlation was found between treatment satisfaction and the EC-17 ( $\mathrm{r}=0.490, \mathrm{p}=0.003)$ at baseline. At followup, a strong correlation was found between treatment satisfaction and the SF-12 PCS ( $\mathrm{r}=0.575, \mathrm{p}=0.000)$. No other significant correlations were found.

At baseline, $69.4 \%$ of the patients reported to have suffered from one or more non-specific symptoms in the past four weeks. These included fatigue (57.3\%), headache (36.0\%) and sleep problems (34.8\%). After four weeks this was $70.4 \%$, with fatigue (53.9\%), headache $(37.5 \%)$ and sleep problems (26.4\%) most often mentioned. A total of 59 patients $(60.2 \%)$ indicated at both measure moments to have suffered from one or more non-specific symptoms in the past four weeks. A moderate negative association was found between the presence of non-specific systems and the SF-12 PCS score $(\mathrm{r}=-0.424, \mathrm{p}=0.005)$ after four

Table 7 Improved scores on individual measures (\% of patients)

\begin{tabular}{llll}
\hline Instrument (n) & Improved score & Same score & Worsened score \\
\hline PAM-13 (47) & $46.8 \%$ & $14.9 \%$ & $38.3 \%$ \\
EC-17 (36) & $47.2 \%$ & $19.4 \%$ & $33.3 \%$ \\
EQ-5D (45) & $40.0 \%$ & $46.7 \%$ & $13.3 \%$ \\
EQ- VAS (47) & $48.9 \%$ & $21.3 \%$ & $29.8 \%$ \\
SF-12 PCS (42) & $57.1 \%$ & $7.1 \%$ & $35.7 \%$ \\
SF-12 MCS (42) & $47.6 \%$ & $7.1 \%$ & $45.2 \%$ \\
GHQ-12 (97) & $47.4 \%$ & $11.3 \%$ & $41.2 \%$ \\
PEI (97) & $22.7 \%$ & $52.6 \%$ & $24.7 \%$ \\
GPE (89) & $41.6 \%$ & $37.1 \%$ & $21.3 \%$ \\
\hline
\end{tabular}

aLower total score on the GHQ reflects improved score. weeks, though at baseline no significant association was found $(r=-0.272, p=0.082)$.

The mean number of reported GP contacts in the past 12 months was 6.9 at baseline. At follow-up, patients reported to have had an average of $1.3 \mathrm{GP}$ contacts in the four weeks between baseline and follow-up. No correlations were found between the number of GP contacts and instrument scores.

\section{Number of questionnaires needed}

Most instruments in our study had a SD between 10-15\% of the instruments' range, resulting in a required sample size of $\mathrm{N}=400$ to detect small differences between baseline and after four weeks [21].

\section{Discussion}

We found high completion rates for all seven instruments, with only a small number of items missing. Total scores for the instruments varied across patients, with the EQ-5D and PEI having a relatively high prevalence of maximum and minimum scores respectively, and most instruments being susceptible for change in the period between baseline and after four weeks. Some strong associations were found between the seven instruments, and between instruments and other measures such as treatment satisfaction and non-specific symptoms, but overall correlations tended to be weak or moderate. Based on our predefined criteria none of the seven instruments seem to stand out in a positive or negative way, and their potential use as PROMs should be studied more elaborately. Finally, the low response rate needs to be considered if PROMs are used in performance measurement systems, because this could lead to selection bias.

Our study is one of the first to explore the use of generic patient-reported outcome measures in primary care. In the US, the Patient-Reported Outcome Measurement Information Systems (PROMIS) aims at the continuing development of patient-reported measures that are comparable across studies and diseases [28]. These measures focus on the domains physical-, mental- and social 
health, and in the current literature on PROMs we also see a focus on quality of life. The present study adds that it explores a broad set of outcome domains (i.e. empowerment, mental health, physical health, general health, enablement and perceived treatment outcome) that all seem to be of importance in primary care.

The present study had a low response compared to recent studies conducted in Dutch general practice $[29,30]$. This low response may indicate selection bias, making it uncertain whether the sample reflected the general practice population. If such a measure were to be used as a performance measure, a low response would have its implications on interpreting the data. In our study we did not send a reminder, because we obtained patients' contact information only after their return of the baseline questionnaire. One potential explanation for the low response rate is the length of the questionnaire. Shortening the measure might result in an increased response in future studies, as has been demonstrated in previous studies [31]. The relatively small size of the study limited the possibility to detect small differences in time or between groups of patients, and significant associations between instruments and other measures. This makes it hard to draw firm conclusions from this study regarding the seven instruments, and replication in larger studies is required with a sample size of at least 400 patients. Despite these limitations, the study provided a number of important leads to the further development of PROMs for adoption in primary care.

Ideally, PROMs are measured before and after a specific intervention. In general practice, however, it is often difficult to determine a clear start and endpoint of treatment. In this study we had two measure moments, both after the consultation with the physician. Therefore the change may reflect effectiveness of interventions, natural course of symptoms, or measurement error. Because continuity of care is one of the hallmarks of general practice, interventions are not limited to one episode of care but cover patient' health needs longitudinally $[4,32]$. The data therefore could still express performance of general practice. Further research is needed to determine if other measure moments than those used in the present study are favourable in primary care.

The seven included instruments were frequently subject of study in previous research, though only limited as outcome measures in the setting of a generic population in general practice.

In our study we found a low responsiveness to change of the EQ-5D, also reflected by a high prevalence of maximum scores at both baseline and after four weeks. Previous studies showed ambiguous results regarding responsiveness of the EQ-5D [33,34]. This might be explained by the different settings in which these studies took place. Our findings suggest that for a generic population visiting the GP other instruments that measure quality of life such as the SF-12 might be more appropriate, though no firm conclusions can be drawn.

The EC-17 specifically focuses on measuring main skills and behaviours needed to effectively manage ones chronic disease. Some of the items of the EC-17 are explicitly targeted at the patients' disease. This resulted in a relatively low number of applicable answers on this instrument, since not all patients in our study population had a disease. The PAM-13 also focuses on chronic patients, though items are targeted at the patients' health instead of the patients' disease, which might explain why this instrument resulted in a higher response rate. This might opt for including the PAM-13 for measuring empowerment, though its validation for a general population in the primary care setting needs be studied.

Previous research that studied the outcome of patient consultations found associations between some of the used instruments and other measures, such as the PEI and the patients' health status [35] and the PEI and treatment satisfaction [18], showed ambiguous results regarding the relation between health status and treatment satisfaction [26,36], and related the presence of non-specific symptoms to emotional distress [20]. In our study we only found a few strong associations, such as that between the GHQ and SF-12 MCS scores, which was to be expected since they both measure mental health, and between treatment satisfaction and the physical component scale of the SF-12. No other strong associations were found between instruments, or with other measures.

This study is to our knowledge one of the first that studies several previously validated questionnaires on different domains as potential PROMs in primary care. It may be used in the further exploration of adapting PROMs in general practice, though our findings are only preliminary results and further research is needed. We think that embedding a short informative measure in the care delivery process where it acts as a feedback tool on the patients' level brings along opportunities. This way the added value for both GP and patient is clear, and it is easier for the GP to act upon this feedback in daily practice if needed. On the other side, embedding PROMs in the care process increases workload for the GP, which needs to be taken into consideration. The potential use of the used instruments as an individual feedback tool in the primary care setting should be studied more elaborately as well. Further research is needed to determine the psychometric properties of previously validated instruments in the current setting of study (i.e. generic primary health care population). Finally, the relation between the studied instruments with relevant clinical measures and the quality of delivered care is a point of interest for future studies. 


\section{Conclusions}

This study showed that several generic instruments on the domains of quality of life, patient empowerment and patient enablement might be fit for use as a PROM in primary care, though further research is needed to study their validity in primary care.

\section{Competing interests}

The authors declare that they have no competing interests.

\section{Authors' contributions}

JW designed the study, was responsible for data collection and data analysis, and wrote the paper. JB and MW designed the study, supervised data-collection and data-analysis, and contributed to the paper. All authors have read and approved the final manuscript.

\section{Acknowledgements}

We thank the patients and general practitioners for their participation

Received: 7 October 2013 Accepted: 17 April 2014

Published: 6 May 2014

\section{References}

1. Dawson J, Doll H, Fitzpatrick R, Jenkinson C, Carr AJ: The routine use of patient reported outcome measures in healthcare settings. BMJ 2010, 340:c186.

2. Fitzpatrick R, Davey C, Buxton MJ, Jones DR: Evaluating patient-based outcome measures for use in clinical trials. Health Technol Assess 1998, 2:i-iv-1-74.

3. Timmins N: NHS goes to the PROMS. BMJ 2008, 336:1464-1465.

4. Allen J, Gay B, Crebolder H, Heyrman J, Svab I, Ram P: The European definitions of the key features of the discipline of general practice: the role of the GP and core competencies. Br J Gen Pract 2002, 52:526-527.

5. Safran DG, Kosinski M, Tarlov AR, Rogers WH, Taira DH, Lieberman N, Ware JE: The Primary Care Assessment Survey: tests of data quality and measurement performance. Med Care 1998, 36:728-739.

6. Grol R, Wensing M, Mainz J, Jung HP, Ferreira P, Hearnshaw H, Hjortdahl P, Olesen F, Reis S, Ribacke M, Szecsenyi J: Patients in Europe evaluate general practice care: an international comparison. Br J Gen Pract 2000, 50:882-887.

7. Wensing M, van Lieshout J, Jung HP, Hermsen J, Rosemann T: The Patients Assessment Chronic Illness Care (PACIC) questionnaire in The Netherlands: a validation study in rural general practice. BMC Health Serv Res 2008, 8:182.

8. Paterson C: Measuring outcomes in primary care: a patient generated measure, MYMOP, compared with the SF-36 health survey. BMJ 1996, 312:1016-1020.

9. Reilly D, Mercer SW, Bikker AP, Harrison T: Outcome related to impact on daily living: preliminary validation of the ORIDL instrument. BMC Health Serv Res 2007, 7:139.

10. Bergner M, Bobbitt RA, Carter WB, Gilson BS: The Sickness Impact Profile: development and final revision of a health status measure. Med Care 1981, 19:787-805

11. Spielberger CD: Manual for the State/Trait Anxiety Inventory (form Y): (Self Evaluation Questionnaire). Palo Alto: Consulting Psychologists Press; 1983.

12. Hibbard JH, Stockard J, Mahoney ER, Tusler M: Development of the Patient Activation Measure (PAM): conceptualizing and measuring activation in patients and consumers. Health Serv Res 2004, 39:1005-1026.

13. Kristjansson E, Tugwell PS, Wilson AJ, Brooks PM, Driedger SM, Gallois C, O'Connor AM, Qualman A, Santesso N, Wale J, Wells GA: Development of the effective musculoskeletal consumer scale. J Rheumatol 2007 34:1392-1400

14. Rabin R, de Charro F: EQ-5D: a measure of health status from the EuroQol Group. Ann Med 2001, 33:337-343.

15. Lamers LM, Stalmeier PF, McDonnell J, Krabbe PF, Van Busschbach JJ: Measuring the quality of life in economic evaluations: the Dutch EQ-5D tariff. Ned Tijdschr Geneeskd 2005, 149:1574-1578.

16. Ware J Jr, Kosinski M, Keller SD: A 12-Item Short-Form Health Survey: construction of scales and preliminary tests of reliability and validity. Med Care 1996, 34:220-233.
17. Goldberg DP, Williams PDPM: A User's Guide to the General Health Questionnaire. Windsor, UK: NFER-NELSON; 1988.

18. Howie JG, Heaney DJ, Maxwell M, Walker JJ: A comparison of a Patient Enablement Instrument (PEI) against two established satisfaction scales as an outcome measure of primary care consultations. Fam Pract 1998, 15:165-171.

19. Kamper SJ, Ostelo RW, Knol DL, Maher CG, De Vet HC, Hancock MJ: Global Perceived Effect scales provided reliable assessments of health transition in people with musculoskeletal disorders, but ratings are strongly influenced by current status. J Clin Epidemiol 2010, 63:760-766. e761.

20. Matalon A, Kotliroff A, Blumberg G, Yaphe J, Kitai E: Non-specific symptoms as clues to changes in emotional well-being. BMC Fam Pract 2011, 12:77.

21. Cohen J: Statistical Power Analysis for the Behavioral Sciences. 2nd edition. Hillsdale, N.J: L. Erlbaum Associates; 1988.

22. Beaton DE, Bombardier C, Katz JN, Wright JG: A taxonomy for responsiveness. J Clin Epidemiol 2001, 54:1204-1217.

23. Evans JD: Straightforward Statistics for the Behavioral Sciences. Pacific Grove: Brooks/Cole; 1996.

24. Streiner DL, Norman GR: Health Measurement Scales: A Practical Guide to their Development and Use. 3rd edition. Oxford, New York: Oxford University Press; 2003.

25. Terwee CB, Bot SD, de Boer MR, van der Windt DA, Knol DL, Dekker J, Bouter LM, de Vet HC: Quality criteria were proposed for measurement properties of health status questionnaires. J Clin Epidemiol 2007, 60:34-42.

26. Chen H, Rosenzweig EB, Gotzkowsky SK, Arneson C, Nelsen AC, Bourge RC: Treatment satisfaction is associated with improved quality of life in patients treated with inhaled treprostinil for pulmonary arterial hypertension. Health Qual Life Outcomes 2013, 11:31.

27. Van der Lucht F, Polder JJ: Towards Better Health: The Dutch 2010 Public Health Status and Forecasts Report, Rijksinstituut voor Volksgezondheid en Milieu RIVM. 2010.

28. Cella D, Riley W, Stone A, Rothrock N, Reeve B, Yount S, Amtmann D, Bode R, Buysse D, Choi S, Cook K, Devellis R, DeWalt D, Fries JF, Gershon R, Hahn EA, Lai JS, Pilkonis P, Revicki D, Rose M, Weinfurt K, Hays R: The Patient-Reported Outcomes Measurement Information System (PROMIS) developed and tested its first wave of adult self-reported health outcome item banks: 2005-2008. J Clin Epidemio/ 2010, 63:1179-1194.

29. Weenink JW, van Lieshout J, Jung HP, Wensing M: Patient Care Teams in treatment of diabetes and chronic heart failure in primary care: an observational networks study. Implement Sci 2011, 6:66.

30. Jansink R, Braspenning J, Keizer E, van der Weijden T, Elwyn G, Grol R: No identifiable $\mathrm{Hb} 1 \mathrm{Ac}$ or lifestyle change after a comprehensive diabetes programme including motivational interviewing: a cluster randomised trial. Scand J Prim Health Care 2013, 31:119-127.

31. Edwards PJ, Roberts I, Clarke MJ, Diguiseppi C, Wentz R, Kwan I, Cooper R, Felix LM, Pratap S: Methods to increase response to postal and electronic questionnaires. Cochrane Database Syst Rev 2009, 3:MR000008.

32. Kringos DS, Boerma WG, Hutchinson A, van der Zee J, Groenewegen PP: The breadth of primary care: a systematic literature review of its core dimensions. BMC Health Serv Res 2010, 10:65.

33. Konig HH, Born A, Gunther O, Matschinger H, Heinrich S, Riedel-Heller SG, Angermeyer MC, Roick C: Validity and responsiveness of the EQ-5D in assessing and valuing health status in patients with anxiety disorders. Health Qual Life Outcomes 2010, 8:47.

34. Brettschneider C, Konig HH, Herzog W, Kaufmann C, Schaefert R, Konnopka A: Validity and responsiveness of the EQ-5D in assessing and valuing health status in patients with somatoform disorders. Health Qual Life Outcomes 2013, 11:3

35. Ozvacic Adzic Z, Katic M, Kern J, Lazic D, Cerovecki Nekic V, Soldo D: Patient, physician, and practice characteristics related to patient enablement in general practice in Croatia: cross-sectional survey study. Croat Med J 2008, 49:813-823.

36. Redekop WK, Koopmanschap MA, Stolk RP, Rutten GEHM, Wolffenbuttel $\mathrm{BHR}$, Niessen LW: Health-related quality of life and treatment satisfaction in Dutch patients with type 2 diabetes. Diabetes Care 2002, 25:458-463.

\section{doi:10.1186/1471-2296-15-88}

Cite this article as: Weenink et al:: Patient reported outcome measures (PROMs) in primary care: an observational pilot study of seven generic instruments. BMC Family Practice 2014 15:88. 